\title{
NRM12 Program
}

\section{International Scientific Advisory Committee}

Journal of Cerebral Blood Flow \& Metabolism (2012) 32, S2; doi:10.1038/jcbfm.2012.72

Anissa Abi-Dargham - New York, USA

Nat Alpert — Boston, USA

Dirk Bender - Aarhus, Denmark

Richard Carson - New Haven, USA

Vin Cunningham - London, UK

Jacques Delforge - Orsay, France

Yu-Shin Ding - New York, USA

Doris Doudet - Vancouver, Canada

Christopher Endres - Baltimore, USA

Lars Farde - Stolkholm, Sweden

Kirk Frey - Ann Arbor, USA

Tony Gee - London, UK

Albert Gjedde - Aarhus, Denmark

Mark Goodman - Atlanta, USA

Roger Gunn — London, UK

Christer Halldin - Stockholm, Sweden

Alexander Hammers - Lyon, France

Karl Herholz - Manchester, UK

Peter Herscovitch - Bethesda, USA

Oliver Howes - London, UK

Robert Innis - Bethesda, USA

Robert Kessler — Nashville, USA

Michael Kilbourn - Ann Arbor, USA

Gitte Knudsen - Copenhagen, Denmark
Bob Koeppe - Ann Arbor, USA

Adriaan Lammertsma - Amsterdam, Netherlands

Andreas Meyer-Lindenberg - Mannheim, Germany

Jean Logan - Upton, USA

Edythe London- Los Angeles, USA

Diana Martinez - New York, USA

Romina Mizrahi - Toronto, Canada

Evan Morris - New Haven, USA

Raj Narendran - Pittsburgh, USA

Ramin Parsey - New York, USA

Julie Price - Pittsburgh, USA

Martin Pomper - Baltimore, USA

Juha Rinne - Turku, Finland

Michio Senda - Kobe, Japan

John Siebyl - New Haven, USA

Mark Slifstein - New York, USA

Vesna Sossi - Vancouver, Canada

Tetsuya Suhara - Chiba, Japan

Hideo Tsukada - Hamamatsu, Japan

Takashi Tsukamoto - Baltimore, USA

Federico Turkheimer - London, UK

Alan Wilson - Toronto, Canada

Karmen Yoder — Indianapolis, USA 